\title{
Labyrinthe
}

$9 \mid 2001$

Numéro 9

\section{Fins et moyens dans le processus institutionnel}

comment les institutions s'émancipent de leur projet initial

\section{Olivier Marty}

\section{OpenEdition}

Journals

Édition électronique

URL : http://journals.openedition.org/labyrinthe/1122

DOI : $10.4000 /$ labyrinthe.1122

ISSN : 1950-6031

Éditeur

Hermann

Édition imprimée

Date de publication : 30 juin 2001

Pagination : 105-107

Référence électronique

Olivier Marty, «Fins et moyens dans le processus institutionnel », Labyrinthe [En ligne], 9 | 2001, mis en ligne le 11 février 2006, consulté le 01 mai 2019. URL : http://journals.openedition.org/labyrinthe/1122 ; DOI : 10.4000/labyrinthe.1122

Ce document a été généré automatiquement le 1 mai 2019.

Propriété intellectuelle 


\title{
Fins et moyens dans le processus institutionnel
}

\author{
Comment les institutions s'émancipent de leur projet initial
}

\author{
Olivier Marty
}

1 On oppose généralement deux paradigmes pour rendre compte de la création des institutions ${ }^{1}$ : d'un côté celui du contrat social et de l'autre celui du marché. D'aucuns soutiennent même que l'explication en termes de marché aurait totalement évincé l'idée de contrat social en ces temps de pensée unique. L'objet de ce texte est de montrer que ces deux paradigmes ne sont pourtant pas incompatibles et qu'ils se complètent au contraire pour expliquer le phénomène institutionnel*.

2 Selon la métaphore du contrat social, les individus libres et pleinement conscients se réunissent et s'accordent pour créer une institution. Chacun fait des concessions quant à ses propres intérêts personnels pour pouvoir s'entendre sur un intérêt commun avec les autres. Dans le droit fil de ce courant,

3 M. Hauriou définit une institution comme « un groupement humain dominé par une idée d'œuvre à accomplir ${ }^{2}$ » - le maintien de l'ordre pour la police, la diffusion du christianisme pour l'Église ou l'accumulation de capital pour l'entreprise. Plus précisément, le processus d'institutionnalisation se décomposerait en cinq phases successives : $1^{\circ}$ une idée d'œuvre est lancée par quelques individus, $2^{\circ}$ cette idée se propage et un groupe de gens s'accorde pour le réaliser, $3^{\circ}$ dans ce groupe s'élève un pouvoir qui s'empare de la domination pour réaliser l'entreprise, $4^{\circ}$ un débat s'engage et débouche bientôt sur une définition des rôles et des statuts, $5^{\circ}$ enfin cette organisation devient une institution après une assez longue durée de rapports pacifiés en son sein. On a donc bien l'idée que les individus s'accordent sur une fin commune, un projet collectif, pour créer une institution.

4 À l'opposé de cette vision, la métaphore du marché renvoie à un ordre spontané émergeant de la multitude d'actions individuelles. Les individus ne se soucieraient que de leurs propres intérêts, passeraient des accords bilatéraux entre eux, et de ces actes épars naîtrait automatiquement un ordre permettant de réguler l'ensemble social. C'est l'idée de la «main invisible» qui permet de passer automatiquement de la recherche de 


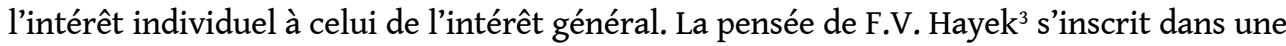
telle vision : il étend l'idée d'un ajustement naturel de l'offre et de la demande sur les marchés pour proposer une vision d'ensemble du fonctionnement des sociétés. Il explique par exemple la génération spontanée de l'institution juridique. Les règles de droit se divisent selon lui en nomos, les traditions et les coutumes, et en thesis, la législation consciente. Seul le nomos importe à ses yeux et celui-ci émerge spontanément au fil des interactions sociales. Il est ensuite conservé ou éliminé selon un processus de sélection naturelle. C'est ainsi la loi du plus fort, la loi du marché, qui guide son élaboration comme sa conservation. À l'inverse de l'idée de contrat social, il n'y a, dans un tel raisonnement, aucun accord conscient sur une fin commune que serait l'institution. Au contraire, celleci apparaît spontanément et est le moyen commun de fins divergentes.

Ces deux explications en termes de contrat social et de marché sont-elles incompatibles? Il paraît possible de les réconcilier en introduisant une dimension temporelle dans l'analyse des institutions. En effet, alors que la création des institutions se comprend par la métaphore d'un contrat social, leur pérennisation s'explique bien mieux par l'idée de marché. Plus précisément, alors qu'une organisation est créée en vue d'une fin sur laquelle s'accordent tous les fondateurs, au fur et à mesure qu'elle s'institutionnalise, la fin est oubliée et elle apparait comme un cadre général au sein duquel s'affrontent les intérêts individuels. Comment rendre compte d'un tel glissement?

6 Les individus qui participent à la naissance d'une institution, les fondateurs, sont essentiellement motivés par l'idée à réaliser. Ils ne voient l'institution qu'ils créent que comme un moyen pour réaliser cette idée. Au contraire, les individus qui entrent dans l'institution alors que celle-ci existe déjà de longue date sont le plus souvent motivés par des avantages parallèles plutôt que par l'idée qu'elle est censée servir. Ainsi, ce sont la plupart du temps des avantages financiers ou symboliques qui décident les individus à entrer au service de telle ou telle institution déjà bien établie, et non pas la cause que celle-ci défend ${ }^{4}$. Et ces individus entrés dans l'institution pour un tout autre motif que l'idée initiale continueront de fonctionner dans cet état d'esprit. C'est-à-dire qu'ils se sentiront bien plus au service de l'institution dont ils profitent qu'à celui de sa cause. Et, une fois arrivés aux postes de commandement, ils chercheront à maintenir ce cadre général qui leur permet de réaliser leurs propres intérêts. Les institutions se détachent donc de leur projet initial parce que les individus qui la composent changent et que, loin d'être motivés par ce projet, la deuxième génération ne cherche plus qu'à réaliser ses propres fins en son sein. L'institution, qui était le moyen d'une fin commune pour les premiers, devient le moyen de fins personnelles divergentes pour les seconds.

7 Le raisonnement peut être poursuivi en indiquant que, dissociées de leur projet initial, les institutions sont aussi prises pour une fin en soi. En effet, alors qu'elles étaient au début clairement vouées à la réalisation d'un objectif - et donc dépendantes de celui-ci à tout instant - le simple fait qu'elles servent par la suite une multitude de fins inavouées et divergentes leur confère une tout autre force. Chaque individu membre aura en effet tendance à vouloir donner de nouvelles raisons d'être à ce cadre d'action si pratique. On essaiera de trouver de nouvelles fins à l'institution pour la légitimer. Ainsi Buchanan ${ }^{5}$ critique l'État Providence car, selon lui, les missions de santé et d'éducation ont été inventées par des gouvernants qui avaient un intérêt politique à ce que l'institution étatique s'élargisse. On aurait ainsi outrepassé le contrat initial avec un État régalien aux fonctions minimales par un glissement qui a conduit à oublier les fins premières et à inventer de nouvelles justifications. L'institution étatique étant renforcée par cette 
évolution qui l'a entrainée à se détacher du projet initial le légitimant pour devenir sa propre fin.

8 Le schéma d'institutionnalisation élaboré par Maurice Hauriou n'est donc valable que pour les institutions les plus jeunes, jusqu'à l'étape $\mathrm{n}^{\circ} 4$. On ne retiendra de sa théorie que l'origine consciente accordée à toute institution : quelques individus cherchent sciemment à réaliser une idée et fondent une institution à cette fin (contrat social). Mais ce contrôle conscient de l'homme sur l'institution disparaît au fur et à mesure que celle-ci vieillit. Les institutions dépassent en effet rapidement des individus qui ne font que passer en leur sein. Au cours d'un processus historique complexe, la fin sur laquelle tous s'étaient accordés est oubliée et on se retrouve dans une situation de marché où plusieurs individus s'affrontent au sein de l'institution pour réaliser leurs propres objectifs. On glisse d'une institution créée par des individus pour servir une fin commune à une institution qui semble vivre pour elle-même et qui abrite des individus aux fins personnelles divergentes. L'institution se pose finalement comme un cadre indépendant qui englobe les individus membres, plutôt qu'elle ne leur sert à réaliser une idée commune. Les paradigmes du contrat social et du marché se complètent donc bien pour former un même cadre explicatif du phénomène institutionnel.

\section{NOTES}

1. Selon P. Rosanvallon, Le Libéralisme, histoire de la notion de marché, Paris, Point politique, Seuil, 1989. L'auteur emploie le terme de « paradigme » (au sens de Kuhn) pour montrer combien ces deux explications renvoient à des visions du monde bien différentes. Il trace même une généalogie des penseurs tenants de chaque bord (généalogie qui pourrait être mise en perspective avec l'opposition entre les rationalistes constructivistes et les rationalistes critiques que développe Hayek.)

*. Ce texte est le résumé d'un mémoire (soutenu à l'IEP de Toulouse en septembre 1999) sur le thème " Les ultra-libéraux face à l'intérêt général, de la théorie à la pratique ". (disponible sur la page : http://www.catallaxia.org/catallaxia/Pepites/ frame_pepites.html, du site www.catallaxia.org).

2. Maurice Hauriou, dans les premières pages de son Traité de droit administratif, écrivait : « une institution sociale est une entreprise dont l'idée domine tellement le personnel des agents qu'elle est devenue pour eux une œuvre à accomplir ».

3. Principalement dans Droit, Législation et Liberté, Quadrige, 1995 et Scientismes et Sciences sociales, Paris, Plon, Agora, 1986.

4. Cf. l'étude d'Olson (La Logique de l'action collective, Paris, PUF, 1978) sur les syndicats, où il démontre que l'adhésion se fait plus par souci d'améliorer sa situation personnelle (par le biais d'une plus grande sécurité ou de relations utiles) que de servir la cause défendue par le syndicat.

5. Cf. J. Buchanan, Les Limites de la liberté, éditions Litec, collection Liberalia, 1992. 